\title{
Sirolimus-based immunosuppression improves outcomes in liver transplantation recipients with hepatocellular carcinoma beyond the Hangzhou criteria
}

\author{
Sunbin Ling ${ }^{1,2 \#}$, Tingting Feng ${ }^{1,2,3 \#}$, Qifan Zhan $^{1,2 \#}$, Xin Duan ${ }^{1,2}$, Guangjiang Jiang ${ }^{1,2}$, Tian Shen ${ }^{1,2}$, \\ Qiaonan Shan ${ }^{1,2}$, Shengjun Xu ${ }^{1,2}$, Qianwei Ye ${ }^{1,2}$, Peng Liu ${ }^{1,2,4}$, Beini Cen ${ }^{2}$, Shusen Zheng ${ }^{1,2}$, Xiao Xu ${ }^{1,2}$ \\ ${ }^{1}$ Department of Hepatobiliary and Pancreatic Surgery, the First Affiliated Hospital, School of Medicine, Zhejiang University, Hangzhou \\ 310003, China; ${ }^{2}$ NHC Key Laboratory of Combined Multi-organ Transplantation, Key Laboratory of the Diagnosis and Treatment of Organ \\ Transplantation, CAMS, Hangzhou 310003, China; ${ }^{3}$ Department of Abdominal Medical Oncology, Zhejiang Cancer Hospital, Hangzhou 310022, \\ China; ${ }^{4}$ Center of Organ Transplantation, the Affiliated Hospital of Qingdao University, Qingdao 266071, China \\ Contributions: (I) Conception and design: S Ling, X Xu; (II) Administrative support: X Xu, S Zheng; (III) Provision of study materials or patients: Q \\ Zhan, X Duan, G Jiang, T Shen; (IV) Collection and assembly of data: T Feng, Q Shan, S Xu, B Cen; (V) Data analysis and interpretation: S Ling, Q \\ Ye, P Liu; (VI) Manuscript writing: All authors; (VII) Final approval of manuscript: All authors. \\ "These authors contributed equally to this work. \\ Correspondence to: Xiao Xu, MD. Department of Hepatobiliary and Pancreatic Surgery, the First Affiliated Hospital, School of Medicine, Zhejiang \\ University, Hangzhou 310003, China. Email: zjxu@zju.edu.cn.
}

Background: The administration of calcineurin inhibitors (CNIs) posttransplant has been implicated as an independent risk factor for the recurrence of hepatocellular carcinoma (HCC) after liver transplantation (LT). The new immunosuppressive agent sirolimus (SRL) acts as a primary immunosuppressant or antitumor agent. In this study we investigated the effect of sirolimus-based immunosuppression compared to CNIs (non-SRL) on the outcomes of LT candidates with HCC.

Methods: We retrospectively analyzed 204 HCC patients who underwent LT in our hospital between January 2, 2014 and December 10, 2017. The median of the follow-up duration of patients was 24.5 months. The patients were divided into a sirolimus (SRL) group (76 patients) and a non-sirolimus (non-SRL) group (128 patients). Patients exceeding the LT criteria were analyzed as subgroups. Disease-free survival (DFS) and overall survival (OS) after tumor recurrence were compared using the Kaplan-Meier method. Univariate and multivariate Cox analyses were used to compare OS between the SRL and non-SRL groups.

Results: The SRL group achieved better OS compared to the non-SRL group, while there was no significant difference in DFS. Subgroup (Milan criteria-based or Hangzhou criteria-based) analyses revealed that patients exceeding, rather than meeting, the Milan or Hangzhou criteria benefited from SRL (exceeding the Milan criteria: $\mathrm{P}=0.002$; exceeding the Hangzhou criteria: $\mathrm{P}<0.001$ ). There was no significant difference in OS between the SRL group and the non-SRL group that met the Milan or Hangzhou criteria.

Conclusions: SRL can improve survival outcomes in LT patients with HCC exceeding the Hangzhou criteria.

Keywords: Sirolimus; liver transplantation (LT); hepatocellular carcinoma (HCC)

Submitted Sep 30, 2019. Accepted for publication Dec 17, 2019.

doi: $10.21037 / \mathrm{atm} .2020 .01 .10$

View this article at: http://dx.doi.org/10.21037/atm.2020.01.10 


\section{Introduction}

Hepatocellular carcinoma (HCC) with a 5 -year survival rate of $14-18 \%$, makes a major contribution to malignancyrelated death worldwide (1). In particular, more than 400,000 people die of HCC every year in China, accounting for over half of all global liver cancer-related deaths (2). Liver transplantation (LT) is one of the most effective treatments for HCC, which removes the entire diseased liver and minimizes tumor recurrence rates. However, the 5 -year tumor recurrence rate posttransplant is $20-57.8 \%$ (3-5), and tumor recurrence posttransplant is associated with a poor prognosis.

In the past two decades, the most effective means to reduce the rate of recurrence posttransplant has been careful screening for transplant recipients. The introduction of the Milan criteria in 1996 facilitated patient selection (6). However, the Milan criteria impose too strict restrictions on tumor size and number, and many HCC patients will lose the chance of LT based on the Milan criteria. Some new selection criteria for liver transplant recipients have been introduced, such as the University of California San Francisco standard (7) and the up-to-seven standard (3). the Hangzhou criteria proposed by our center introduced the biological characteristics and pathological features of tumors for the first time as the selection criteria for liver transplant recipients (4).

Calcineurin inhibitors (CNIs), such as tacrolimus (Tac) and cyclosporine A (CsA), are the most common immunosuppressants for solid organ transplantation, including LT (8). They can significantly prevent acute rejection. However, at the same time, they also have obvious side effects, such as cardiovascular complications, kidney toxicity, and diabetes (9). In addition, the administration of CNIs posttransplant has been implicated as an independent risk factor for the recurrence of HCC after LT (10). The new immunosuppressive agent mTOR inhibitors such as sirolimus (SRL) and everolimus have been shown to have antiproliferative and antiangiogenic pharmacological effects in a variety of solid tumors $(11,12)$. However, it is still not clear whether mTOR inhibitors have a potential benefit for antitumor activity in HCC transplant patients, and the population who benefits remains controversial (13). Therefore, in the present study, we retrospectively analyzed the effects of SRL-based immunosuppressive regimens posttransplant on the recurrence and survival of 204 HCC patients who underwent LT between January 2, 2014 and December 10, 2017, to provide suggestions for HCC liver cancer transplant recipients.

\section{Methods}

\section{Study population and data sources}

In our study, we collected data on 204 HCC patients who underwent liver transplantation at the First Affiliated Hospital of Zhejiang University between January 2, 2014 and December 10, 2017 (Figure 1). All the patients received deceased donor liver transplantation. The median of the follow-up duration of patients was 24.5 months. The patients' data were obtained from the China Liver Transplant Registry (CLTR) database and our center's database. The patients were divided into two groups according to the presence or absence of SRL treatment: the SRL group and the non-SRL group. The following inclusion criteria were applied: pathologically confirmed HCC; and a complete postoperative follow-up record. The following exclusion criteria were applied: patients initially using SRL less than 30 days before tumor recurrence; patients with a survival time of less than 80 days; patients with noncontinuous drug treatment; patients who died of noncancer causes; and patients with multiple primary malignant tumors.

The variables examined were age, sex, HCC LT criteria (Milan or Hangzhou criteria), the Edmondson grade, the number of tumor nodules, vascular invasion, size of the major nodule, the level of preoperative AFP expression, treatment before LT [transarterial chemoembolization (TACE) and radiofrequency ablation (RFA)], the preoperative model of end-stage liver disease (MELD) score, the presence of a capsule complete and the HBV infection status.

\section{Immunosuppressive protocol}

In all patients, baliximab $(20 \mathrm{mg})$ was administered within 2 hours before operation and in the forth day after operation. Methylprednisolone $(500 \mathrm{mg}$ ) was intraoperative administered. Then, Tac/CyA + mycophenolate-based immunosuppressive protocol was performed in the early period after operation. In the SRL group, SRL was typically administered 30-45 days after transplantation because it may delay wound healing. The blood concentration of SRL was stable at $4-10 \mathrm{ng} / \mathrm{mL}$. The dose of CNI was reduced to half at the start of SRL and withdrawn when target levels of SRL were reached. In the control group, tacrolimus/ 


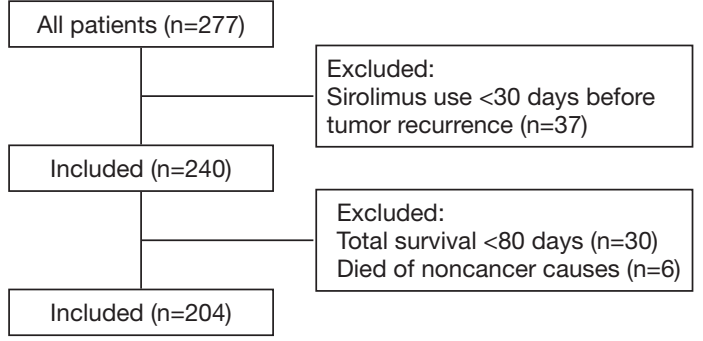

Figure 1 Diagram of patient selection.

cyclosporine A was kept administered and the dosage was adjusted according to liver function and concentration of the blood immunosuppressant. The mycophenolate was used all the time in both groups.

\section{Statistical analysis}

Statistical analysis was performed using the Statistical Package for Social Sciences (SPSS 23.0 IBM Corporation, Armonk, NY). All $\mathrm{P}$ values were two-tailed, and significance was defined as $\mathrm{P}<0.05$. Follow-up after transplantation was defined as the time from transplantation to death or the time of the last follow-up. A chi-square test was used for the statistical analysis of categorical variables. A $t$-test was used for the statistical analysis of continuous variables. Overall survival (OS) and disease-free survival (DFS) were the primary outcomes and they were computed using the Kaplan-Meier method, and a log-rank test was used to assess differences between curves. All the factors with $\mathrm{P}<0.05$ in the Cox univariate analysis were also analyzed in the multivariate analysis using the Cox proportional hazards model.

\section{Results}

\section{Patient characteristics}

A total of 204 liver transplant recipients with HCC were examined in this study. There were 76 patients in the SRL group and 128 patients in the non-SRL group. No significant difference in demographics was found between the two groups (Table 1). Notably, HBV-infected patients accounted for $89 \%$ of all the patients in our study: $84.8 \%$ in the SRL group and $84.8 \%$ in the non-SRL group.

\section{Survival analysis in all patients}

The median DFS and OS times in both groups were determined (Figure 2A,B). The 1- and 3-year DFS rates of the SRL group were $76.3 \%$ and $65.7 \%$, respectively, while in the non-SRL group, the 1- and 3-year DFS rates were $68.0 \%$ and $66.4 \%$, respectively. No significant difference was found between the two groups $(\mathrm{P}=0.755)$. The 1 - and 3 -year OS rates of the SRL group were $97.4 \%$ and $85.5 \%$, respectively, while in the non-SRL group, the 1- and 3-year OS rates were $82.0 \%$ and $71.9 \%$, respectively. The SRL group had a better prognosis than the non-SRL group $(\mathrm{P}<0.001)$.

Univariate and multivariate Cox regression analyses of the risk factors for overall survival of all patients showed that the use of SRL was an independent protective factor of prognosis (Table 2), while the number of tumor nodules, the level of preoperative AFP expression and vascular invasion were independent risk factors for prognosis.

\section{Effect of SRL on the survival of HCC patients after LT based on LT criteria}

In the patients fulfilling the Milan criteria, there were 26 patients in the SRL group and 51 patients in the nonSRL group. No significant difference in OS was found between the two groups $(\mathrm{P}=0.799)$ (Figure $3 A)$. There were 39 patients and 73 patients who fulfilled the Hangzhou criteria in the SRL group and the non-SRL group, respectively. Similarly, no significant difference in OS was found between the two groups $(\mathrm{P}=0.978)$ (Figure 3B).

There were 50 patients and 77 patients exceeding the Milan criteria in the SRL group and the non-SRL group, respectively. The median OS of patients exceeding the Milan criteria was 35.5 months in the non-SRL group, while the median OS of patients in the SRL group was not determined. We found that patients exceeding the Milan criteria in the SRL group experienced longer OS than those in the non-SRL group at each time point examined $(\mathrm{P}<0.001)$ (Figure $3 C)$. Similarly, among the 92 liver transplant recipients exceeding the Hangzhou criteria, the SRL group experienced longer OS than those in the non-SRL group at each time point examined $(\mathrm{P}<0.001)$ (Figure 3D). The median OS of patients exceeding the Hangzhou criteria was 20.3 months in the non-SRL group, while the median OS of patients in the SRL group was not determined.

However, no significant difference in DFS was observed between the SRL and non-SRL groups regardless of whether the patients fulfilled or exceeded both the Milan and Hangzhou criteria after LT (Figure S1). The effect of 
Table 1 Characteristics of the patients

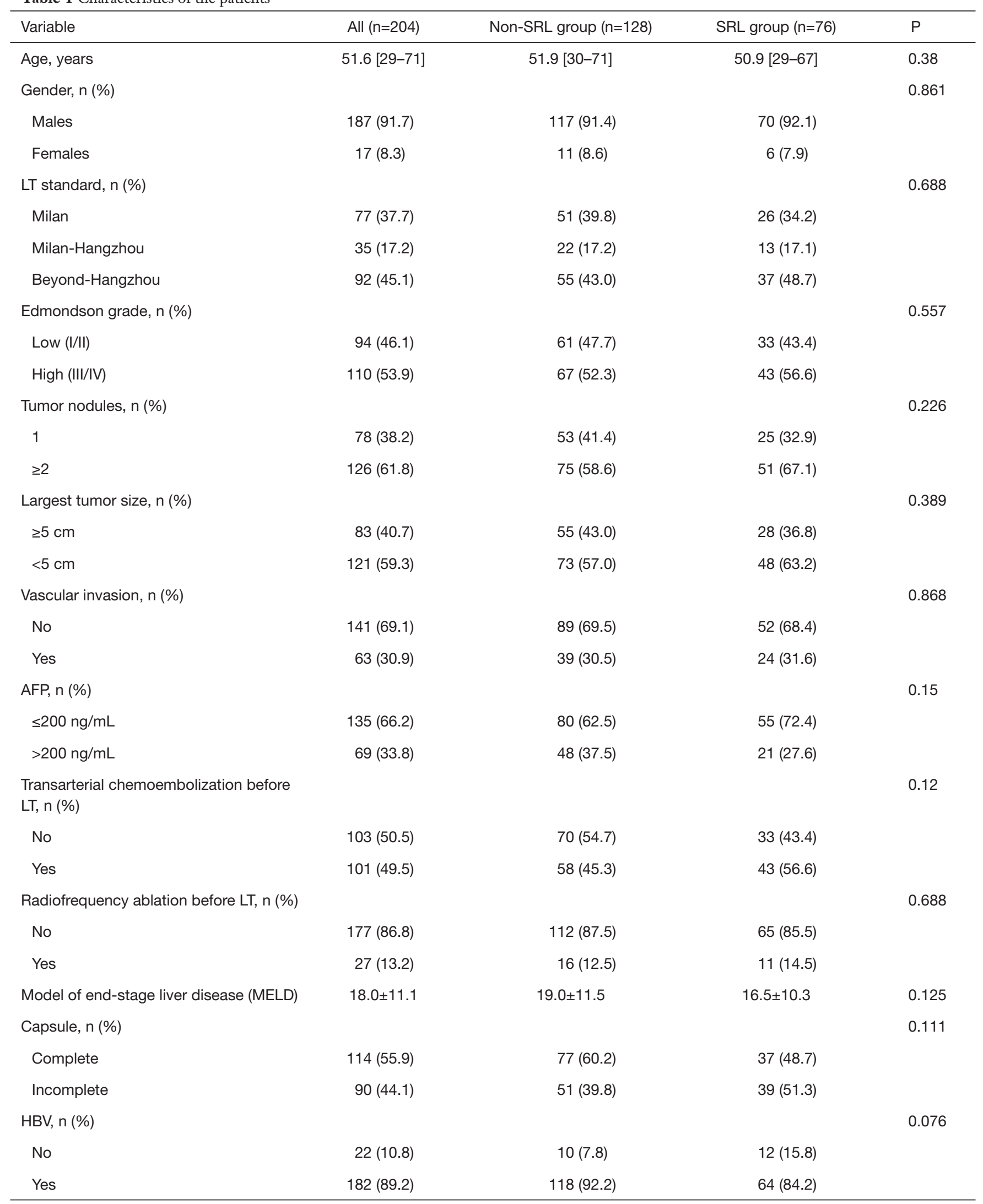



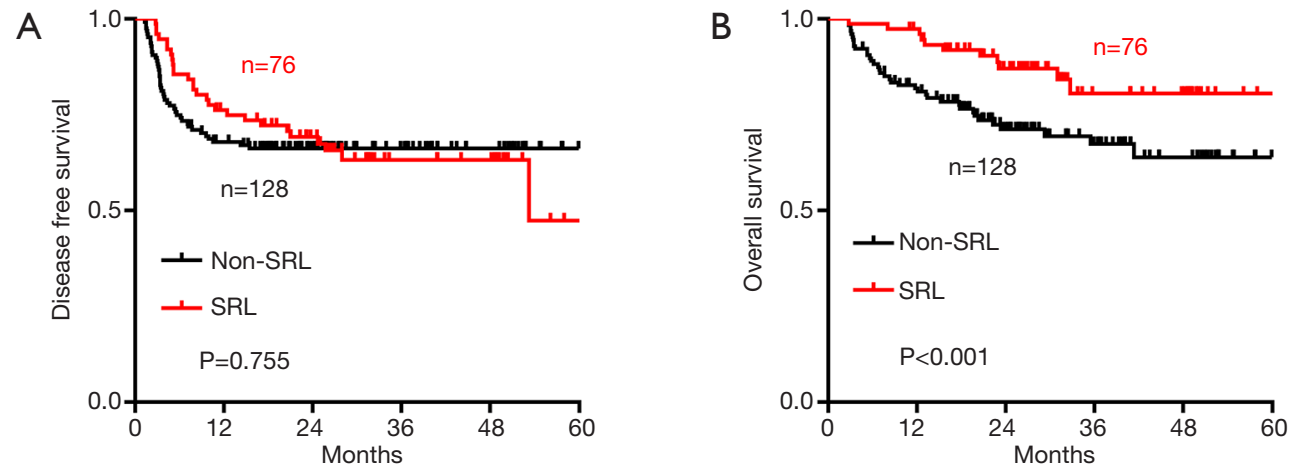

Figure 2 Effect of sirolimus (SRL) on the survival of hepatocellular carcinoma (HCC) patients after liver transplantation (LT). The diseasefree survival (DFS) (A) and overall survival (OS) (B) were analyzed between the non-SRL and SRL groups of HCC patients after LT (Kaplan-Meier, log-rank test).

Table 2 Univariate and multivariate Cox regression analysis of risk factors for overall survival of the all patients

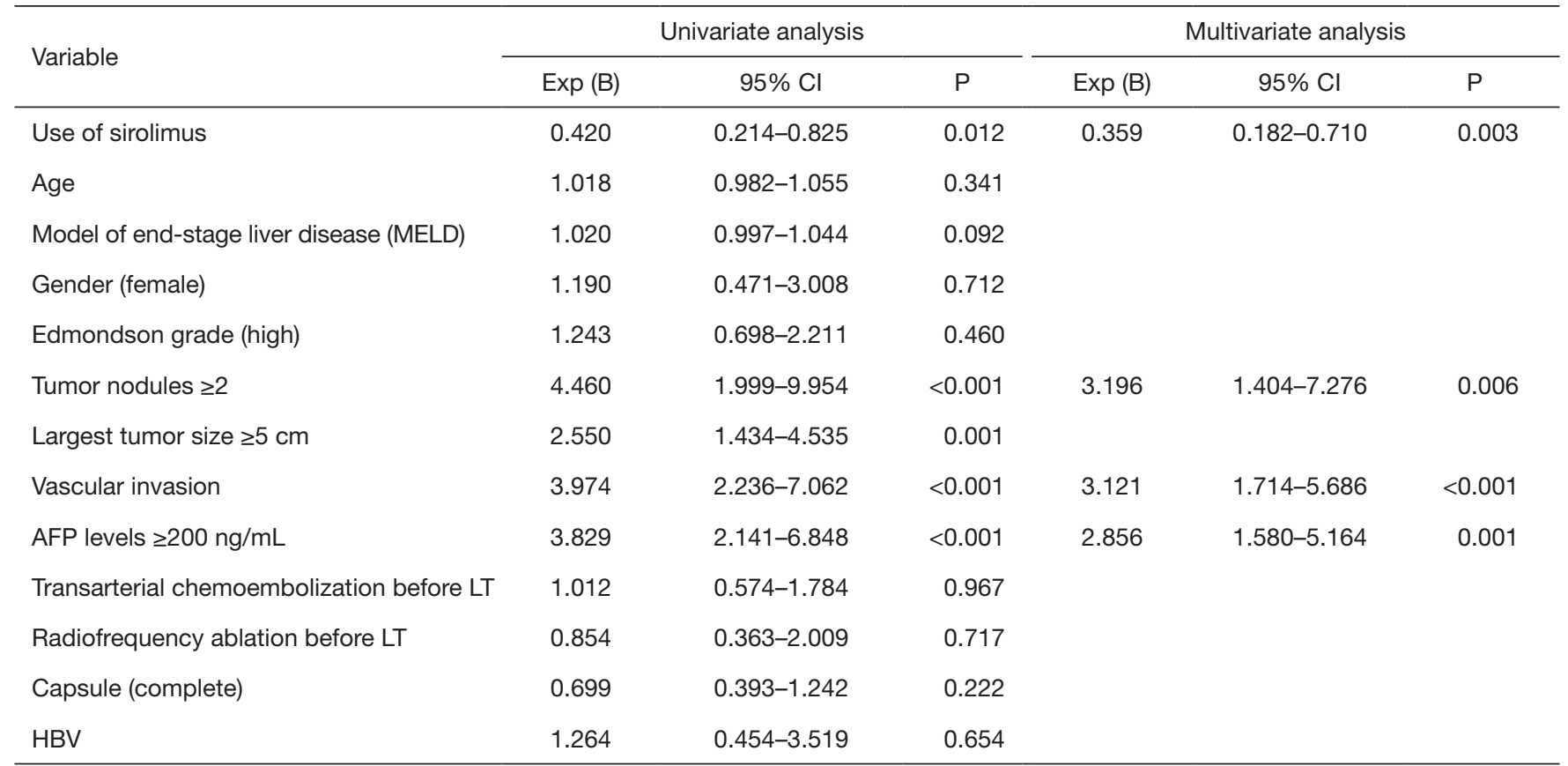

SRL on the survival of HCC patients who exceeded the Milan criteria but fulfilled the Hangzhou criteria after LT was also explored. DFS (Figure $S 2 A$ ) and OS (Figure $S 2 B$ ) were not significantly different between the non-SRL and SRL groups of HCC patients after LT.

\section{Survival analysis in patients who exceeded the Milan or Hangzhou criteria after $L T$}

Univariate and multivariate Cox regression analyses of risk factors for overall survival of the patients who exceeded the Milan criteria after LT showed that the use of SRL was an independent protective factor of prognosis $(\mathrm{P}=0.003)$, while a preoperative AFP level $\geq 200 \mathrm{ng} / \mathrm{mL}(\mathrm{P}<0.001)$ and vascular invasion $(\mathrm{P}=0.011)$ were identified as independent risk factors for prognosis (Table 3).

Univariate and multivariate Cox regression analyses of risk factors for the overall survival of patients who exceeded the Hangzhou criteria showed that the use of SRL was an independent protective factor of prognosis $(\mathrm{P}=0.002)$, while a preoperative AFP level $\geq 200 \mathrm{ng} / \mathrm{mL}$ $(\mathrm{P}=0.001)$ was identified as an independent risk factor for 

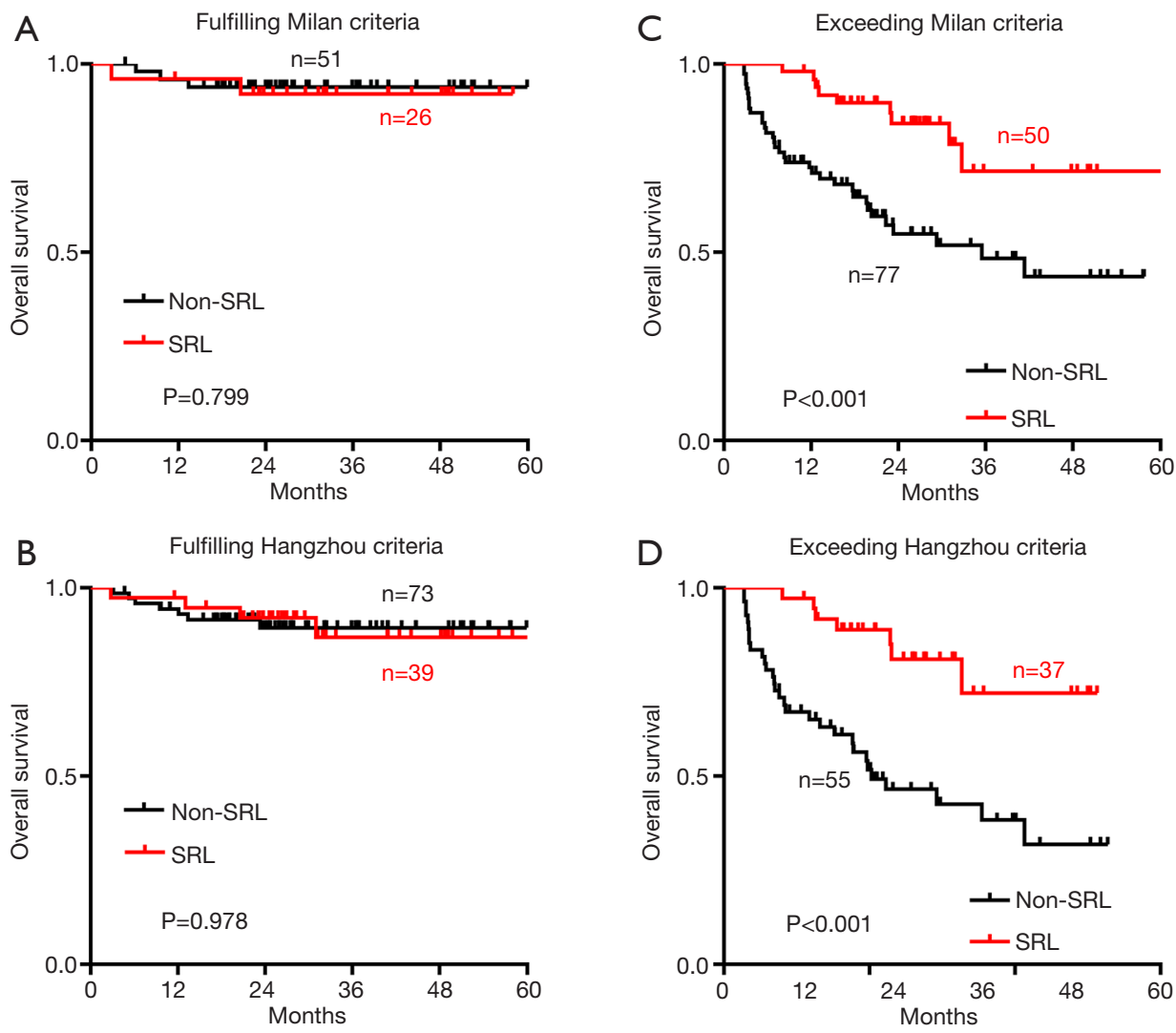

Figure 3 Effect of sirolimus (SRL) on the survival of hepatocellular carcinoma (HCC) patients after liver transplantation (LT). The overall survival (OS) were analyzed between the non-SRL or SRL groups of HCC patients after liver transplantation (LT) based on Milan criteria $(\mathrm{A}, \mathrm{C})$ or Hangzhou criteria (B,D) (Kaplan-Meier, log-rank test).

Table 3 Univariate and multivariate Cox regression analysis of risk factors for overall survival of the patients exceeding Milan criteria

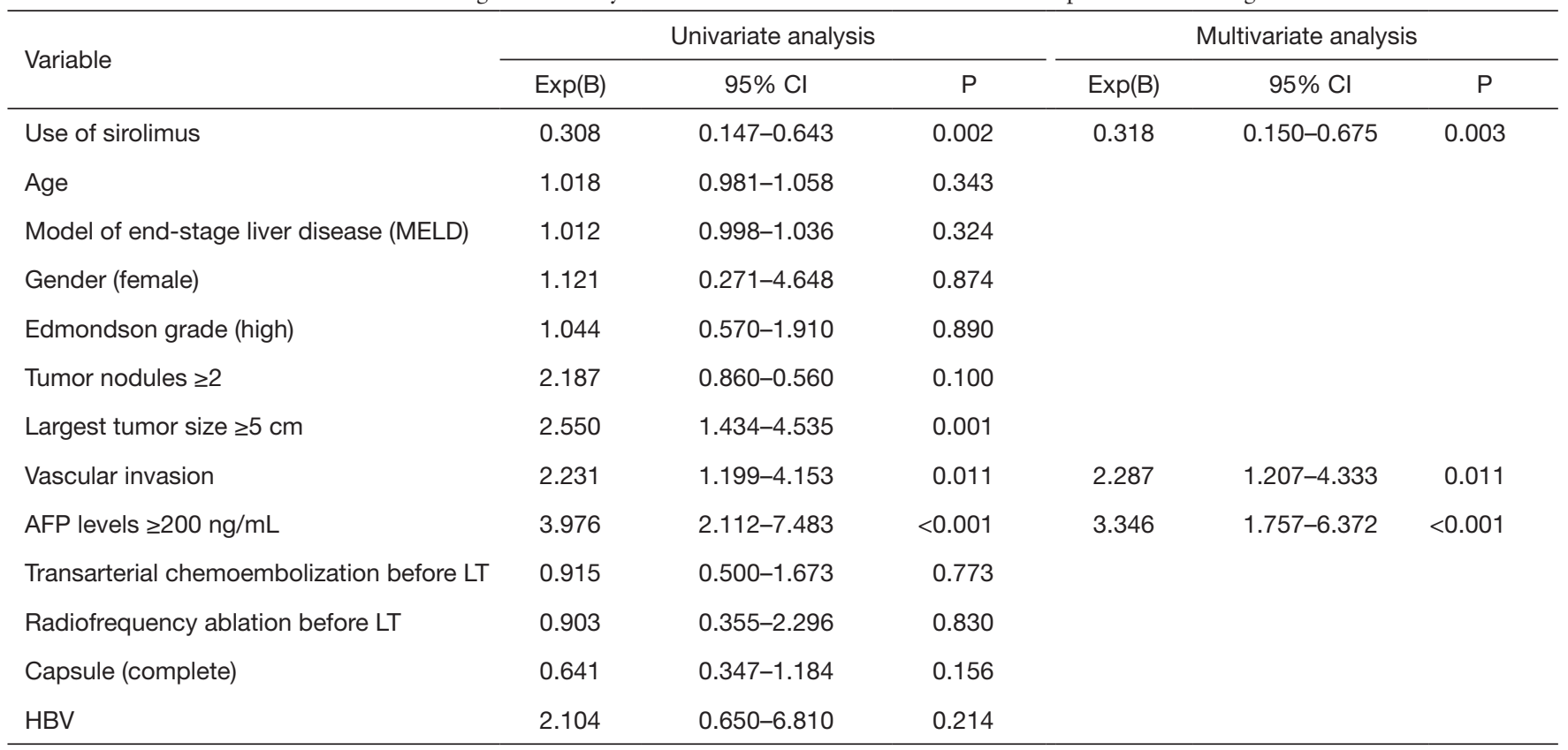


Table 4 Univariate and multivariate Cox regression analysis of risk factors for overall survival of the patients exceeding Hangzhou criteria

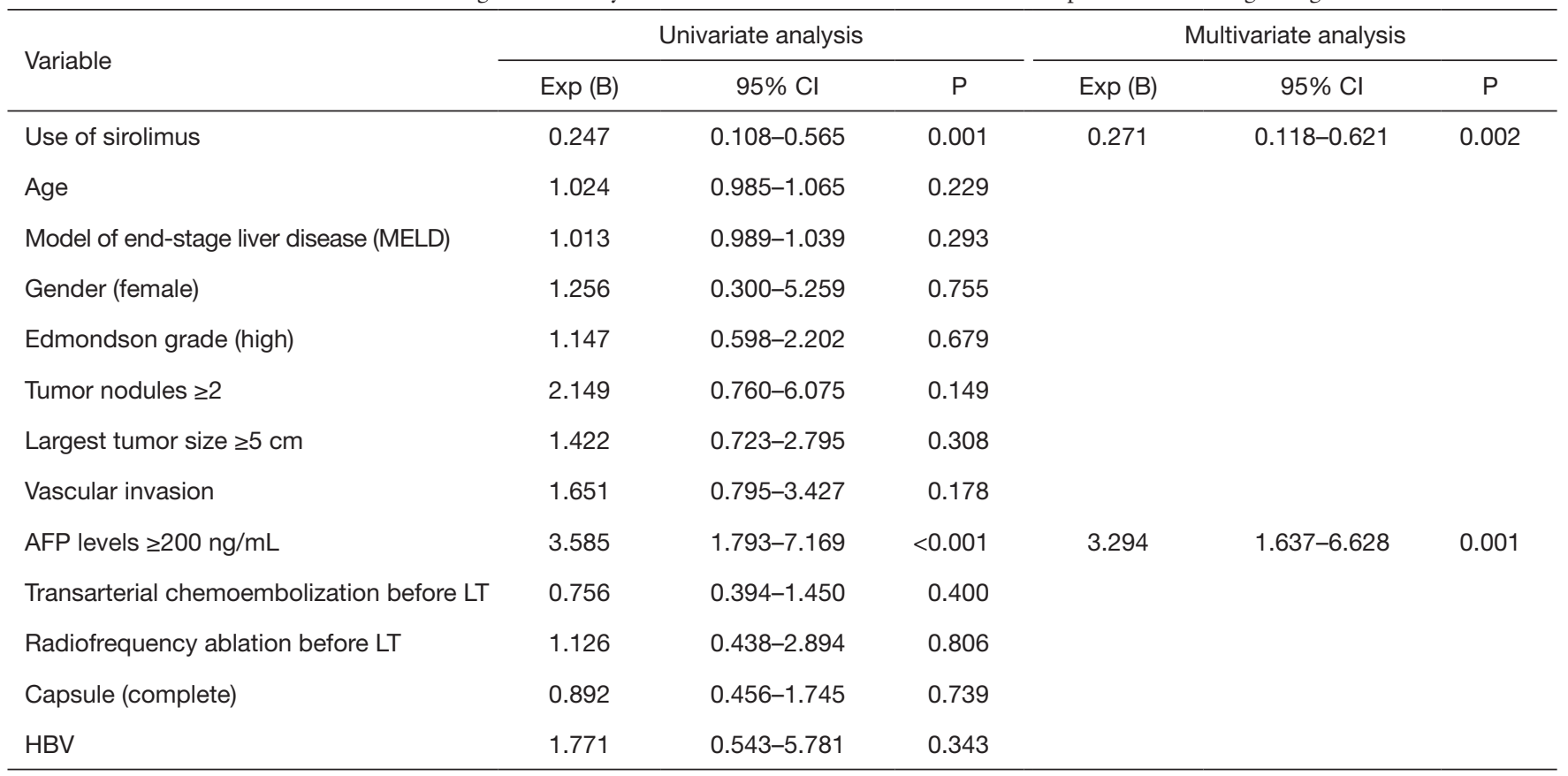

prognosis (Table 4).

The characteristics of the patients in the subgroups described above are displayed in Table S1 and Table S2. It is worth noting that the proportion of patients with a preoperative AFP level $\geq 200 \mathrm{ng} / \mathrm{mL}$ in the SRL group was significantly higher than that in the non-SRL group.

\section{Discussion}

Postoperative tumor recurrence is the main factor affecting the survival of HCC patients with LT. In our study, we found that SRL improved the survival outcome of liver transplant recipients with HCC. The role of SRL in HCC patients with LT is controversial (13-15). Previous studies have suggested that SRL is not beneficial in reducing mortality (16) because of an insufficient sample size. However, Yanik et al. reported that SRL did not appear to be beneficial in reducing all-cause mortality among 3,936 HCC liver recipients (14). We believe that although the sample size was large, all patients who met the Milan criteria were included, which may have resulted in a negative result. Interestingly, another study found that SRL was associated with significant reduction in the risk of death for liver recipients transplanted for HCC, compared to those that remained on tacrolimus or cyclosporine" (15).

Although accumulating evidence suggests that HCC patients with LT may benefit from SRL (15,17-19), the population who may benefit from SRL is controversial. In our study, the subgroup survival analysis found that HCC patients with LT both beyond the Milan criteria and beyond the Hangzhou criteria benefited from SRL. Similarly, the other two studies from China also suggest that SRL may improve the OS of HCC liver transplant recipients beyond the transplant criteria (UCSF or the Milan criteria) $(18,19)$. A phase 3 randomized controlled trial reported that SRL in liver transplant recipients with HCC did not improve longterm RFS beyond 5 years, in part, due to the increasing rate of SRL discontinuation after 3 years, exceeding $35 \%$ by 5 years. However, RFS and OS benefits were evident in the first 3 to 5 years, especially in low-risk (fulfilled the Milan criteria) patients (13). We hypothesize that the ethnic and etiological differences may be responsible for this finding. Approximately $90 \%$ of patients in our study had hepatitis $\mathrm{B}$, and $95.1 \%$ of the patients in the SiLVER study were Caucasian. Hepatitis $\mathrm{C}$ and nonalcoholic hepatitis are the main causes of HCC in Caucasians.

Although Zhou et al. (19) also reported a survival benefit of postoperative SRL in patients with HCC beyond the Milan criteria, the factors of included in the survival analysis analyzed only the influence of the Child-Pugh-Turcotte Score, microvascular invasion and the immunosuppressive protocol was assessed on prognosis and lacked information 
on important variables, such as the Edmondson grade, the number of tumor nodules, the preoperative AFP level, and the treatment protocol before LT. Furthermore, the Milan criteria may be too strict for screening for liver transplant recipients. The Hangzhou criteria, which are more suitable for HCC patients with hepatitis B in China, break through the limitation of a tumor diameter of $5 \mathrm{~cm}$ and add AFP expression and the tumor histological grade as conditional restrictions, expanding the indications for LT for HCC patients (4). Interestingly, although the OS time of the SRL group was prolonged compared to that of the nonSRL group in patients exceeding the Milan or Hangzhou criteria, no significant difference in OS was found between the SRL and non-SRL groups in patients who exceeded the Milan criteria but met the Hangzhou criteria. However, the sample size of patients in this group is small, and further large-sample studies are needed.

Similar to previous studies $(14,20)$, SRL did not improve postoperative recurrence in all liver transplant patients with HCC. However, in patients exceeding the Hangzhou criteria, we observed that the SRL group experienced longer DFS than the non-SRL group, although no significant difference was detected.

There are certain limitations to our study. This study was a retrospective study; therefore, selection bias is inevitable. We need prospective studies and high-level randomized controlled trials to confirm the conclusions of this study in the future.

\section{Acknowledgments}

Funding: This work was supported in part by funding from the National Science and Technology Major Project of China (No. 2017ZX10203205) and the Project of Medical and Health Technology Program in Zhejiang Province (No. 2019RC152).

\section{Footnote}

Conflicts of Interest: The authors have no conflicts of interest to declare.

Ethical Statement: The authors are accountable for all aspects of the work in ensuring that questions related to the accuracy or integrity of any part of the work are appropriately investigated and resolved. The study protocol was approved by the Research Ethics Committee of the First Affiliated Hospital of Zhejiang University, and informed consent was obtained from all participants. No donor livers were harvested from executed prisoners.

\section{References}

1. Siegel RL, Miller KD, Jemal A. Cancer statistics, 2019. CA Cancer J Clin 2019;69:7-34.

2. Ding C, Fu X, Zhou Y, et al. Disease burden of liver cancer in China from 1997 to 2016: an observational study based on the Global Burden of Diseases. BMJ Open 2019;9:e25613.

3. Mazzaferro V, Llovet JM, Miceli R, et al. Predicting survival after liver transplantation in patients with hepatocellular carcinoma beyond the Milan criteria: a retrospective, exploratory analysis. Lancet Oncol 2009;10:35-43.

4. $\mathrm{Xu} X, \mathrm{Lu} \mathrm{D}$, Ling Q, et al. Liver transplantation for hepatocellular carcinoma beyond the Milan criteria. Gut 2016;65:1035-41.

5. Duvoux C, Roudot-Thoraval F, Decaens T, et al. Liver transplantation for hepatocellular carcinoma: a model including alpha-fetoprotein improves the performance of Milan criteria. Gastroenterology 2012;143:986-94 e3; quiz e14-5.

6. Mazzaferro V, Regalia E, Doci R, et al. Liver transplantation for the treatment of small hepatocellular carcinomas in patients with cirrhosis. $\mathrm{N}$ Engl J Med 1996;334:693-9.

7. Mazzaferro V, Bhoori S, Sposito C, et al. Milan criteria in liver transplantation for hepatocellular carcinoma: an evidence-based analysis of 15 years of experience. Liver Transpl 2011;17 Suppl 2:S44-57.

8. Dhanasekaran R. Management of Immunosuppression in Liver Transplantation. Clin Liver Dis 2017;21:337-53.

9. Tsay AJ, Eisen HJ. mTOR inhibitors vs calcineurin inhibitors: A Catch-22-preventing nephrotoxicity or acute allograft rejection after heart transplantation. Am J Transplant 2019;19:2967-8.

10. Lerut J, Iesari S, Foguenne M, et al. Hepatocellular cancer and recurrence after liver transplantation: what about the impact of immunosuppression? Transl Gastroenterol Hepatol 2017;2:80.

11. De Simone P, Fagiuoli S, Cescon M, et al. Use of Everolimus in Liver Transplantation: Recommendations From a Working Group. Transplantation 2017;101:239-51.

12. Jeng LB, Lee SG, Soin AS, et al. Efficacy and safety of everolimus with reduced tacrolimus in living-donor liver transplant recipients: 12 -month results of a randomized 
multicenter study. Am J Transplant 2018;18:1435-46.

13. Geissler EK, Schnitzbauer AA, Zulke C, et al. Sirolimus Use in Liver Transplant Recipients With Hepatocellular Carcinoma: A Randomized, Multicenter, Open-Label Phase 3 Trial. Transplantation 2016;100:116-25.

14. Yanik EL, Chinnakotla S, Gustafson SK, et al. Effects of maintenance immunosuppression with sirolimus after liver transplant for hepatocellular carcinoma. Liver Transpl 2016;22:627-34.

15. Toso C, Merani S, Bigam DL, et al. Sirolimus-based immunosuppression is associated with increased survival after liver transplantation for hepatocellular carcinoma. Hepatology 2010;51:1237-43.

16. Zimmerman MA, Trotter JF, Wachs M, et al. Sirolimusbased immunosuppression following liver transplantation for hepatocellular carcinoma. Liver Transpl 2008;14:633-8.

17. Grigg SE, Sarri GL, Gow PJ, et al. Systematic review

Cite this article as: Ling S, Feng T, Zhan Q, Duan X, Jiang G, Shen T, Shan Q, Xu S, Ye Q, Liu P, Cen B, Zheng S, Xu X. Sirolimus-based immunosuppression improves outcomes in liver transplantation recipients with hepatocellular carcinoma beyond the Hangzhou criteria. Ann Transl Med 2020;8(4):80. doi:10.21037/atm.2020.01.10 with meta-analysis: sirolimus- or everolimus-based immunosuppression following liver transplantation for hepatocellular carcinoma. Aliment Pharmacol Ther 2019;49:1260-73.

18. Zhou L, Pan LC, Zheng YG, et al. Novel strategy of sirolimus plus thymalfasin and huaier granule on tumor recurrence of hepatocellular carcinoma beyond the UCSF criteria following liver transplantation: A single center experience. Oncol Lett 2018;16:4407-17.

19. Zhou J, Wang Z, Wu ZQ, et al. Sirolimus-based immunosuppression therapy in liver transplantation for patients with hepatocellular carcinoma exceeding the Milan criteria. Transplant Proc 2008;40:3548-53.

20. Xu SL, Zhang YC, Wang GY, et al. Survival analysis of sirolimus-based immunosuppression in liver transplantation in patients with hepatocellular carcinoma. Clin Res Hepatol Gastroenterol 2016;40:674-81. 

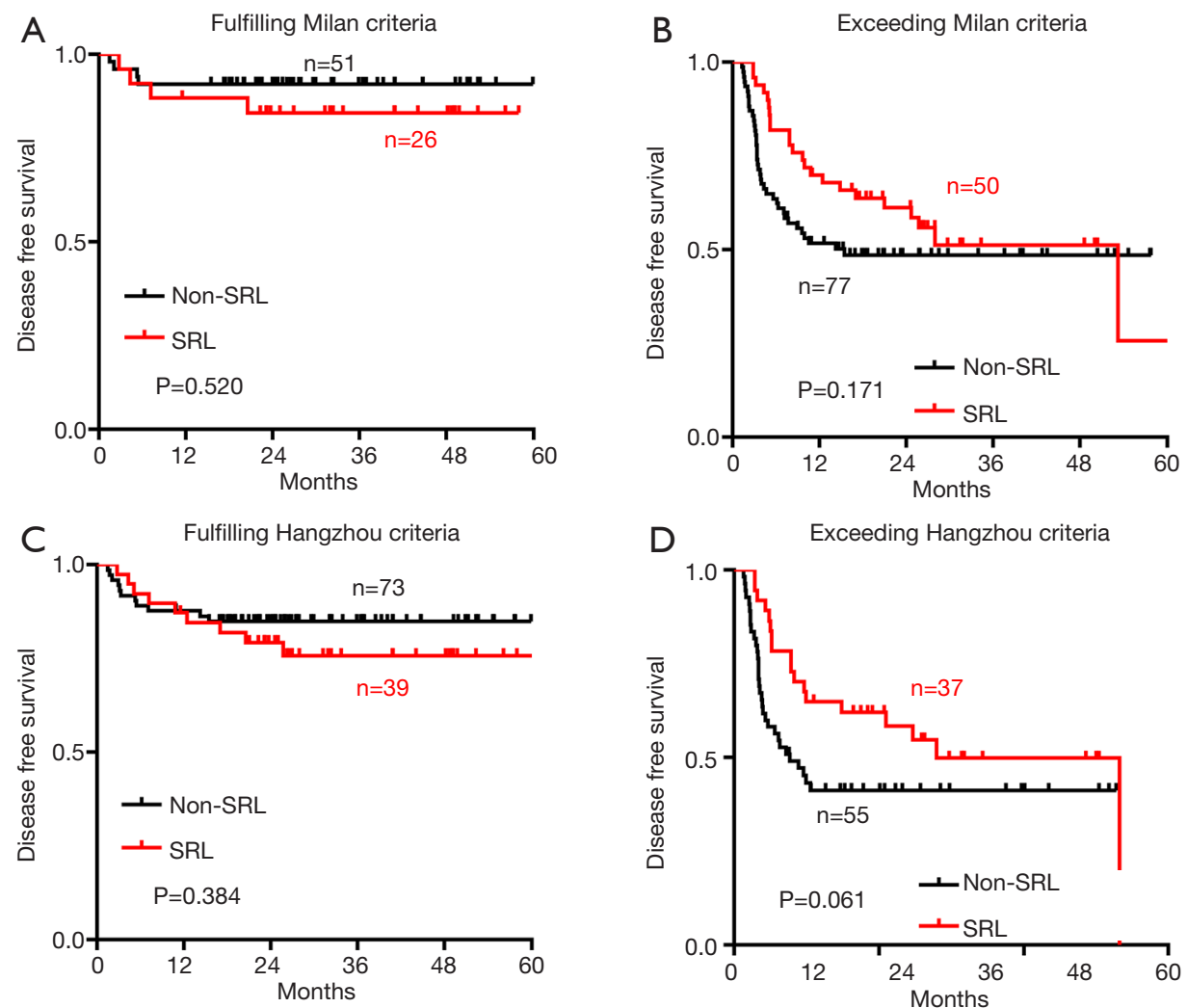

Figure S1 Effect of sirolimus (SRL) on the survival of hepatocellular carcinoma (HCC) patients after liver transplantation (LT) based on LT criteria. The disease free survival (DFS) were analyzed between the non-SRL or SRL groups of HCC patients after LT based on Milan criteria (A,B) or Hangzhou criteria (C,D) (Kaplan-Meier, log-rank test).
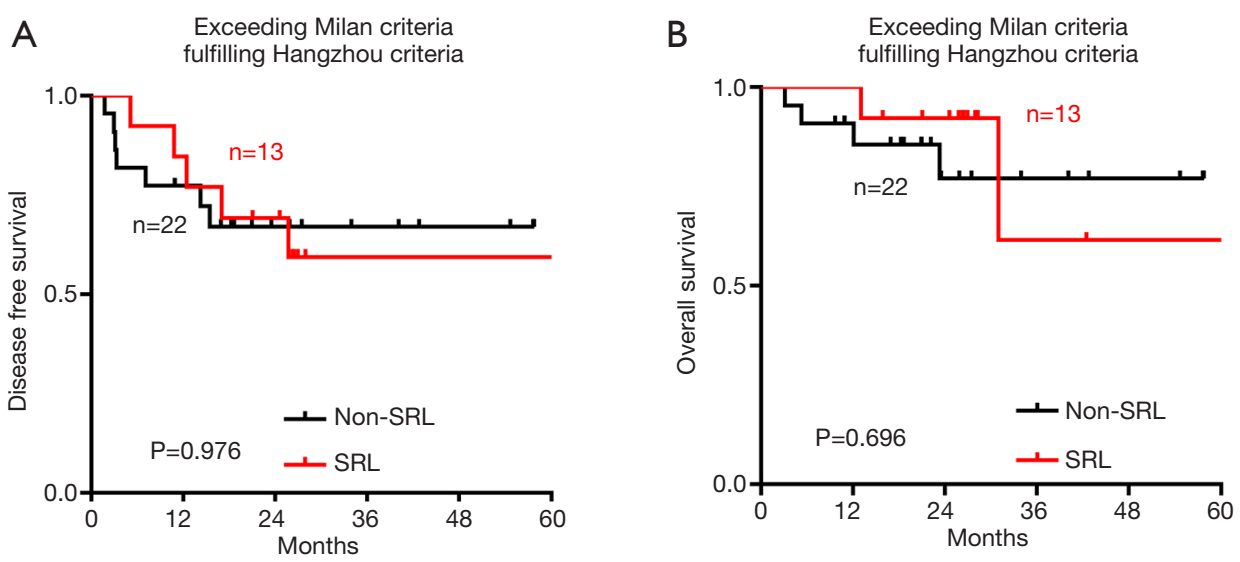

Figure S2 Effect of sirolimus (SRL) on the survival of hepatocellular carcinoma (HCC) patients exceeding Milan criteria but fulfilling Hangzhou criteria after liver transplantation (LT). The disease free survival (DFS) (A) and overall survival (OS) (B) were analyzed between the non-SRL and SRL groups of HCC patients after LT (Kaplan-Meier, log-rank test). 
Table S1 Characteristics of the patients who exceeding Milan criteria

\begin{tabular}{|c|c|c|c|c|}
\hline Variable & All $(n=127)$ & $\begin{array}{c}\text { Non-SRL } \\
\text { group }(n=77)\end{array}$ & $\begin{array}{l}\text { SRL group } \\
\qquad(\mathrm{n}=50)\end{array}$ & $P$ \\
\hline Age & 51.7 [29-71] & $52.31[33-71]$ & 50.7 [29-67] & 0.296 \\
\hline Gender & & & & 0.366 \\
\hline Males & $122(96.1)$ & 73 (94.8) & 49 (98.0) & \\
\hline Females & $5(3.9)$ & $4(5.2)$ & $1(2.0)$ & \\
\hline Edmondson grad & & & & 0.301 \\
\hline Low (I/II) & $58(45.7)$ & $38(49.4)$ & $20(40)$ & \\
\hline High (III/IV) & $69(54.3)$ & $39(50.6)$ & $30(60)$ & \\
\hline Tumor nodules & & & & 0.597 \\
\hline 1 & 25 (19.7) & 14 (18.2) & $11(22.0)$ & \\
\hline$\geq 2$ & 102 (80.3) & 63 (81.8) & 39 (78.0) & \\
\hline $\begin{array}{l}\text { Largest tumor } \\
\text { size }\end{array}$ & & & & 0.128 \\
\hline$\geq 5 \mathrm{~cm}$ & $53(41.7)$ & $28(36.4)$ & $25(50.0)$ & \\
\hline$<5 \mathrm{~cm}$ & 74 (58.3) & 49 (63.6) & $25(50.0)$ & \\
\hline Vascular invasion & & & & 0.77 \\
\hline No & $64(50.4)$ & $38(49.4)$ & $26(52.0)$ & \\
\hline Yes & 63 (49.6) & $39(50.6)$ & $24(48.0)$ & \\
\hline AFP & & & & 0.006 \\
\hline$\leq 200 \mathrm{ng} / \mathrm{mL}$ & 75 (59.1) & $38(49.4)$ & $37(74.0)$ & \\
\hline$>200 \mathrm{ng} / \mathrm{mL}$ & $52(40.9)$ & $39(50.6)$ & $13(26.0)$ & \\
\hline TACE before LT & & & & 0.504 \\
\hline No & $58(45.7)$ & $37(48.1)$ & $21(42.0)$ & \\
\hline Yes & 69 (54.3) & $40(51.9)$ & $29(58.0)$ & \\
\hline RFA before LT & & & & 0.777 \\
\hline No & $113(89.0)$ & $69(89.6)$ & $44(88.0)$ & \\
\hline Yes & $14(11.0)$ & $8(10.4)$ & $6(12.0)$ & \\
\hline MELD & $19.8 \pm 11.8$ & $22.18 \pm 12.7$ & $16.0 \pm 9.6$ & 0.003 \\
\hline Capsule & & & & 0.475 \\
\hline Complete & $56(44.1)$ & $32(41.6)$ & $24(48.0)$ & \\
\hline Incomplete & 71 (55.9) & $45(58.4)$ & $26(52.0)$ & \\
\hline HBV & & & & 0.352 \\
\hline No & 16 (12.6) & 8 (10.4) & 8 (16.0) & \\
\hline Yes & $111(874)$ & 69 (89.6) & $42(84.0)$ & \\
\hline
\end{tabular}

Table S2 Characteristics of the patients exceeding Hangzhou criteria

\begin{tabular}{|c|c|c|c|c|}
\hline Variable & All $(n=92)$ & $\begin{array}{c}\text { Non-SRL } \\
\text { group }(n=55)\end{array}$ & $\begin{array}{l}\text { SRL group } \\
\quad(n=37)\end{array}$ & $\mathrm{P}$ \\
\hline Age & $51.5[29-71]$ & $52.8[33-71]$ & 49.4 [29-63] & 0.079 \\
\hline Gender, n (\%) & & & & 0.526 \\
\hline Males & $88(95.7)$ & $36(97.3)$ & $52(94.5)$ & \\
\hline Females & $4(4.3)$ & $1(2.7)$ & $3(5.5)$ & \\
\hline \multicolumn{2}{|c|}{ Edmondson grade, n (\%) } & & & 0.16 \\
\hline Low (I/II) & $43(46.7)$ & $29(52.7)$ & $14(37.8)$ & \\
\hline High (III/IV) & $49(53.3)$ & $26(47.3)$ & $23(62.2)$ & \\
\hline \multicolumn{2}{|c|}{ Tumor nodules, n (\%) } & & & 0.524 \\
\hline 1 & $17(18.5)$ & $9(16.4)$ & $8(21.6)$ & \\
\hline$\geq 2$ & $75(81.5)$ & $46(83.6)$ & $29(78.4)$ & \\
\hline \multicolumn{2}{|c|}{ Largest tumor size, n (\%) } & & & 0.086 \\
\hline$\geq 5 \mathrm{~cm}$ & $35(38.0)$ & $17(30.9)$ & $18(48.6)$ & \\
\hline$<5 \mathrm{~cm}$ & $57(62.0)$ & $38(69.1)$ & $19(51.4)$ & \\
\hline \multicolumn{2}{|c|}{ Vascular invasion, n (\%) } & & & 0.541 \\
\hline No & $29(31.5)$ & $16(29.1)$ & $13(35.1)$ & \\
\hline Yes & $63(68.5)$ & $39(70.9)$ & $24(64.9)$ & \\
\hline AFP, n (\%) & & & & 0.037 \\
\hline$\leq 200 \mathrm{ng} / \mathrm{mL}$ & $50(54.3)$ & $25(45.5)$ & $25(67.6)$ & \\
\hline$>200 \mathrm{ng} / \mathrm{mL}$ & $42(45.7)$ & $30(54.5)$ & $12(32.4)$ & \\
\hline \multicolumn{2}{|c|}{ TACE before LT, n (\%) } & & & 0.834 \\
\hline No & $41(44.6)$ & $25(45.5)$ & $16(43.2)$ & \\
\hline Yes & $51(55.4)$ & $30(54.5)$ & $21(56.8)$ & \\
\hline \multicolumn{2}{|c|}{ RFA before LT, n (\%) } & & & 0.785 \\
\hline No & $83(90.2)$ & $50(90.9)$ & $5(9.1)$ & \\
\hline Yes & $9(9.8)$ & $33(89.2)$ & $4(10.8)$ & \\
\hline MELD & $19.54 \pm 12.3$ & $21.2 \pm 13.1$ & $17.08 \pm 10.5$ & 0.1 \\
\hline Capsule, n (\%) & & & & 0.372 \\
\hline Complete & $47(51.5)$ & $26(47.3)$ & $29(52.7)$ & \\
\hline Incomplete & 45 (48.9) & $21(56.8)$ & $16(43.2)$ & \\
\hline HBV, n (\%) & & & & 0.459 \\
\hline No & $12(13.0)$ & $6(10.9)$ & $6(16.2)$ & \\
\hline Yes & $80(87.0)$ & 49 (89.1) & $31(83.8)$ & \\
\hline
\end{tabular}

